\title{
GENERALIZED POSITIVE LINEAR FUNCTIONALS ON A BANACH ALGEBRA WITH AN INVOLUTION ${ }^{1}$
}

\author{
PARFENY P. SAWOROTNOW
}

\begin{abstract}
Let $A$ be a proper $H^{*}$-algebra and let $B$ be a Banach *-algebra with an identity. A linear mapping $\varphi: B \rightarrow A$ is called a positive $A$-functional if $\sum_{i, j} a_{i}^{*} \varphi\left(x_{i}^{*} x_{j}\right) a_{j}$ is positive for all $x_{1}$, $x_{2}, \cdots, x_{n} \in B$ and $a_{1}, a_{2}, \cdots, a_{n} \in A$. It is shown that for each positive $A$-functional $\varphi$ there exists a ${ }^{*}$-representation $T$ of $B$ by $A$-linear operators on a Hilbert module $H$ such that $\varphi(x)=$ $\left(f_{0}, T x f_{0}\right)$ for all $x \in B$ and some $f_{0} \in H$. If $B$ is of the form $B=$ $\left\{\lambda e+x \mid \lambda\right.$ complex, $e$ is the (abstract) identity, $\left.x \in L^{1}(G)\right\}$ for some locally compact group $G$ then $\varphi$ has the form $\varphi(\lambda e+x)=\lambda \varphi(e)+$ $\int_{\alpha} x(t) p(t) d t$ for some generalized ( $A$-valued) positive definite function $p$ on $G, x \in L^{1}(G)$.
\end{abstract}

1. The present work is a continuation of the study of Hilbert modules [6], [7]. In the previous paper [7] we generalized the theorem which states that each positive definite function on a group $G$ is of the form $p(t)=$ $\left(U_{t} f_{0}, f_{0}\right)$ for some unitary representation $U$ of $G$, where $f_{0}$ is some member of the Hilbert space on which $U$ acts. In this paper we will generalize the concept of a positive linear functional on a $*$-algebra and will prove that each generalized positive functional $\varphi$ on a Banach algebra $B$ is of the form $\varphi(x)=\left(f_{0}, T x f_{0}\right)$ for some *-representation $x \rightarrow T x$ of $B$ by $A$ linear operators on some Hilbert module.

Applying this result to a group algebra we shall derive an integral representation of the generalized positive linear functional on $L^{1}(G)$ in terms of an $H^{*}$-algebra valued positive definite function on $G$. In this way we will establish generalizations of Theorem 2 in $\$ 17$ and Theorem 2 in $\S 30$ of [5].

2. Let $A$ be a proper $H^{*}$-algebra [1] and let $\tau(A)=\{x y \mid x, y \in A\}$ be its trace-class [8]. It was shown in [8] that $\tau(A)$ is a Banach algebra with respect to some norm $\tau()$ which is related to the norm || of $A$ by the identity " $\tau\left(a^{*} a\right)=|a|^{2}, a \in A$ ". There is a partial ordering defined on

Presented to the Society, April 9, 1971; received by the editors November 25, 1970. AMS 1970 subject classifications. Primary 22D20, 46K15, 46H25; Secondary 46K10, 43A35.

Key words and phrases. Hilbert module, $H^{*}$-algebra, Banach *-algebra, group algebra, *-representation.

1 This research was supported by NSF Grant GP-11118. 
$\tau(A)$ by the requirement that $a \geqq 0$ if $a=b^{*} b$ for some $b \in A$. Also there is a trace $\operatorname{tr}$ defined on $\tau(A)$ such that $\operatorname{tr} a=\tau(a)$ if $a \geqq 0$ and $\operatorname{tr}\left(x y^{*}\right)=$ $\operatorname{tr}\left(y^{*} x\right)=(x, y)$ for all $x, y \in A$ (here $($.$) denotes the scalar product on$ $A)$. For further details on $\tau(A)$ the reader is referred to [8] and [9].

A right module $H$ over $A$ is called a Hilbert $A$-module if there exists a $\tau(A)$-valued function ( , ) on $H \times H$ having the following properties:

(i) If $f, g, h \in H$ and $a \in A$ then $(f+g, h)=(f, h)+(g, h),(f, g)^{*}=$ $(g, f),(f, g a)=(f, g) a,(f, f) \geqq 0$ and $|\operatorname{tr}(f, g)|^{2} \leqq \tau(f, f) \tau(g, g)$.

(ii) $(f, f)=0$ if and only if $f=0$.

(iii) $H$ is complete in the norm $\|f\|=(\tau(f, f))^{1 / 2}$.

The function (, ) is called generalized scalar product. There is a linear structure on $H$ such that $H$ is an ordinary Hilbert space with respect to the scalar product $[f, g]=\operatorname{tr}(g, f)$. An $A$-linear operator on $H$ is an additive mapping $T: H \rightarrow H$ such that $T(f a)=(T f) a$ for all $f \in H, a \in A$; $T$ is bounded in $\|T f\| \leqq M\|f\|$ for some $M \geqq 0$ and all $f \in H$. Each bounded $A$-linear operator $T$ is linear and its adjoint $T^{*}$ has the property that $(T f, g)=\left(f, T^{*} g\right)$ for all $f, g \in H$.

3. Let $B$ be a Banach algebra with the identity $e$ and an involution $x \rightarrow x^{*}$ such that $\left|x^{*}\right|=|x|$ for all $x \in B$ and let $A$ be a proper $H^{*}$ algebra (| | denotes the norm for each algebra).

Definition. A linear mapping $\varphi: B \rightarrow A$ is called a positive $A$-functional if $\sum_{i, j} a_{i}^{*} \varphi\left(x_{i}^{*} x_{j}\right) a_{j} \geqq 0$ for all $x_{1}, x_{2}, \cdots, x_{n} \in B$ and $a_{1}, a_{2}, \cdots$, $a_{n} \in A$.

LeMma 1. If $\varphi$ is a positive A-functional on $B$ then $\varphi\left(x^{*}\right)=\varphi(x)^{*}$ for all $x \in B$.

Proof. If $a \in A$ then the mapping $x \rightarrow \operatorname{tr}\left(a^{*} \varphi(x) a\right)$ is a positive linear functional on $B$ and so $\operatorname{tr}\left(a^{*} \varphi\left(x^{*}\right) a\right)=(\operatorname{tr})^{-}\left(a^{*} \varphi(x) a\right)$ for all $x \in B$, as it was shown on p. 96 of [3]. ${ }^{2}$

Now let $a, b \in A$. Then

$$
\begin{aligned}
4\left(\varphi\left(x^{*}\right) a . b\right)= & 4 \operatorname{tr} b^{*} \varphi\left(x^{*}\right) a \\
= & \operatorname{tr}(a+b)^{*} \varphi\left(x^{*}\right)(a+b)-\operatorname{tr}(a-b)^{*} \varphi\left(x^{*}\right)(a-b) \\
& +i \operatorname{tr}(a+i b)^{*} \varphi\left(x^{*}\right)(a+i b) \\
& -i \operatorname{tr}(a-i b)^{*} \varphi\left(x^{*}\right)(a-i b) \\
= & (\operatorname{tr})^{-}\left[(a+b)^{*} \varphi(x)(a+b)\right]-(\operatorname{tr})^{-}\left[(a-b)^{*} \varphi(x)(a-b)\right] \\
& +i(\operatorname{tr})^{-}\left[(a+i b)^{*} \varphi(x)(a+i b)\right. \\
& -i(\operatorname{tr})^{-}\left[(a-i b)^{*} \varphi(x)(a-i b)\right] \\
= & 4(\operatorname{tr})^{-}\left(a^{*} \varphi(x) b\right)=4 \operatorname{tr}(\varphi(x) b)^{*} a=4(a \cdot \varphi(x) b) .
\end{aligned}
$$

Thus $\varphi\left(x^{*}\right)=\varphi(x)^{*}$ since $a, b$ were arbitrary.

${ }^{2}$ Here and below $(\operatorname{tr})^{-}(\cdots)$ denotes the complex-conjugate of $\operatorname{tr}(\cdots)$. 
Let $H$ be a Hilbert $A$-module, let $x \rightarrow T x$ be a representation of $B$ by bounded $A$-linear operators on $H$ ( $T$ is a homomorphism such that $T e=I$ and $T x^{*}=T^{*} x$ for all $x \in B$ ) and let $f_{0} \in H$. Then $\varphi(x)=$ $\left(f_{0}, T x f_{0}\right)$ is a positive $A$-functional on $B$ :

$\sum_{i, j} a_{i}^{*} \varphi\left(x_{i}^{*} x_{j}\right) a_{j}=\sum_{i, j} a_{i}^{*}\left(f_{0}, T x_{i}^{*} T x_{j} f_{0}\right) a_{j}=\left(\sum_{i} T x_{i} f_{0} a_{i}, \sum_{i} T x_{i} f_{0} a_{i}\right) \geqq 0$.

The converse is also true as it is stated in Theorem 1. A *-representation $T$ of $B$ is said to be regular if $T x(f)=0$ for all $x \in B$ implies $f=0$ (in the terminology of Naimark [5, $\$ 29]: T$ has no degenerate subrepresentations).

THEOREM 1. For each positive A-functional $\varphi$ on a Banach algebra $B$ with an isometric involution (and an identity) there exists a Hilbert $A$-module $H$, a regular *-representation $x \rightarrow T x$ by bounded A-linear operators on $H$ and $f_{0} \in H$ such that $\varphi(x)=\left(f_{0}, T x f_{0}\right)$ for all $x \in B$.

Proof. Let $K$ be the set of all formal expressions $f=\sum_{i=1}^{n} x_{i} a_{i}$ with $x_{i} \in B, a_{i} \in A$; if $g=\sum y_{i} b_{\imath}$ with $y_{i} \in B, b_{i} \in A$ define $(f, g)=$ $\sum_{i, j} a_{i}^{*} \varphi\left(x_{i}^{*} y_{i}\right) b_{j}$. Then it is easy to see that (, ) has all the properties of a generalized scalar product, except that $(f, f)$ may be zero without $f$ being a zero expression. We define $\|f\|=(\tau(f, f))^{1 / 2}=(\operatorname{tr}(f, f))^{1 / 2}$. Then \|\| is a seminorm on $K$ and one can show as in Theorem 2 of [6] that $\tau(f, g) \leqq\|f\| \cdot\|g\|$ for all $f, g \in K$.

Let $\mathfrak{N}=\{f \in K \mid(f, f)=0\}$; then the last inequality implies that $(f, g)=0$ for all $f \in \mathfrak{N}, g \in K$. Also $\mathfrak{N}$ is an $A$-submodule of $K$ (since $\tau(f a, f a)=\tau\left((f, f) a a^{*}\right) \leqq \tau(f, f) \cdot \tau\left(a a^{*}\right)$ if $\left.a \in A\right)$. Let $H^{\prime}=K / \mathfrak{N}$ and let $H$ be the completion of $H^{\prime}$ with respect to the norm of $H^{\prime}$ which is induced by \|\| (we will denote this norm also by \|\| ). Then $H$ is a Hilbert module.

For each $x \in B$ we define an operator $T^{\prime} x$ on $K$ by setting $T^{\prime} x(f)=$ $T^{\prime} x\left(\sum_{i} x_{i} a_{i}\right)=\sum_{i} x x_{i} a_{i}$; then $T^{\prime} x$ is $A$-linear and we shall show that $\left\|T^{\prime} x(f)\right\| \leqq|x| \cdot\|f\|$ for all $f \in K$. This inequality will imply both that $T^{\prime} x$ induces some operator $T x$ on $H^{\prime}$ and that this operator $T x$ is bounded.

So let $f=\sum x_{i} a_{i}$ in $K$ be fixed. The linear functional

$$
\psi(y)=\operatorname{tr} \sum_{i, j} a_{i}^{*} \varphi\left(x_{i}^{*} y x_{j}\right) a_{j}
$$

on $B$ is positive and so it follows from the Section 4 in $\S 10$ of [5] that $|\psi(y)| \leqq|y| \psi(e)$ for all $y \in B$. Taking $y=x^{*} x$ we have:

$$
\begin{aligned}
\left\|T^{\prime} x(f)\right\|^{2} & =\operatorname{tr}\left(T^{\prime} x f, T^{\prime} x f\right)=\operatorname{tr} \sum_{i, j} a_{i}^{*} \varphi\left(x_{i}^{*} x^{*} x x_{j}\right) a_{j}=\psi\left(x^{*} x\right) \\
& \leqq\left|x^{*} x\right| \cdot \psi(e) \leqq\left|x^{*}\right| \cdot|x| \cdot \operatorname{tr} \sum_{i, j} a_{i}^{*} \varphi\left(x_{i}^{*} x_{i}\right) a_{j}=|x|^{2} \cdot\|f\|^{2} .
\end{aligned}
$$

Thus $\left\|T^{\prime} x f\right\| \leqq|x| \cdot\|f\|$ for all $f \in K$ and so $T^{\prime} x$ induces a bounded 
$A$-linear operator $T x$ on $H$. We define $f_{0}=\lim _{n} e e^{n}$, where $e$ is the identity of $B$ and $e^{n}$ is as in the proof of Theorem 1 of [7] ( $p(1)$ should be replaced by $\varphi(e)$ in the condition " $p(1) e=e p(1)=0 \cdots$. $)$.

4. If the algebra $B$ has no identity then we can adjoin it to $B$ and consider the algebra $B_{e}=\{\lambda e+x \mid x \in B, \lambda$ complex $\}$ as it was done, for example, on p. 25 of [4] (or on p. 59 of [3]). We extend the involution to $B_{e}$ by setting $(\lambda e+x)^{*}=\bar{\lambda} e+x^{*}$ and consider the norm $\|\lambda e+x\|=$ $|\lambda|+\|x\|$.

Definition. A positive $A$-functional on $B$ is a mapping $\varphi: B \rightarrow A$ such that there exists a positive $A$-functional $\varphi^{\prime}$ on $B_{e}$ whose restriction to $B$ coincides with $\varphi$.

This definition enables us to apply Theorem 1 to a group algebra in order to obtain a generalization of Theorem 2 in $\$ 30$ of [5], which establishes the correspondence between positive definite functions defined on a topological group and (extendable) positive linear functionals defined on the group algebra.

We will need a few lemmas.

LEMMA 2. Let $H$ be a Hilbert module over a proper $H^{*}$-algebra $A$ and let $T$ be a right centralizer [8] on $A$. Then there exists a bounded linear operator $T^{\prime}$ on $H$ such that $T(f, g)=\left(T^{\prime} f, g\right)$ for all $f, g \in H$.

Proof. For a fixed $f \in H$ the mapping $b: g \rightarrow T(f, g)$ is a bounded $A$-linear functional on $H(\tau(T(f, g)) \leqq\|T\| \cdot \tau(f, g) \leqq\|T\| \cdot\|f\| \cdot\|g\|$ [9]). Thus [6, Theorem 3] there exists $z_{f} \in H$ such that $T(f, g)=\left(z_{f}, g\right)$ for all $g \in H$ and $\|b\|=\left\|z_{f}\right\| \leqq\|T\| \cdot\|f\|$. We define $T^{\prime} f=z_{f} \cdots$.

Now let $(S, \mu)$ be a measurable space and let $h(s)$ be a mapping of $S$ into a Hilbert module $H$ such that the $\tau(A)$-valued function $t(s)=$ $(g, h(s))$ is Pettis integrable for each $g \in H$. This in turn means that the scalar valued function $\varphi_{m}(s)=m(g, h(s))$ is Lebesgue integrable for each $m \in \tau(A)^{*}$ and there exists a member $(P) \int(g, h(s)) d s$ of $\tau(A)$ such that $m\left((P) \int(g, h(s)) d s\right)=\int m(g, h(s)) d \mu(s)$ for all $m \in \tau(A)^{*}$. This condition could be restated as follows [9]: for each right centralizer $T$ on $A$ the mapping $s \rightarrow \operatorname{tr} T(g, h(s))$ is Lebesgue integrable and there exists (P) $\int(g, h(s)) d s \in \tau(A)$ such that

$$
\operatorname{tr} T\left((P) \int(g, h(s)) d s\right)=\int \operatorname{tr} T(g, h(s)) d \mu(s) \quad \text { for all } T \in R(A) .
$$

Definition. We shall say that an $H$-valued function $h(s)$ is $P$-integrable if $(g, h(s))$ is Pettis integrable for all $g \in H$ and there exists $P(h) \in H$ such that $(g, P(h))=(P) \int(g, h(s)) d s$ for all $g \in H$. 
It was shown in [6] that $H$ has also a structure of a Hilbert space with respect to the (ordinary) scalar product $[f, g]=\operatorname{tr}(g, f)$. Therefore one may speak about the Pettis integral of an $H$-valued function. It turns out that both integrals coincide:

Lemma 3. A Hilbert module valued function $h(s)$ is P-integrable if and only if it is Pettis integrable; also $P(h)=(P) \int h(s) d s$.

Proof. Taking $T$ to be the identity operator we see at once that each $P$-integrable function is Pettis integrable.

Conversely let $h(s)$ be Pettis integrable. Then for each $g \in H$ and $T \in R(A)=\tau(A)^{*}$ [9] the function $\xi(s)=\operatorname{tr} T(g, h(s))=\operatorname{tr}\left(T_{g}^{\prime}, h(s)\right)$ is Lebesgue integrable and

$$
\begin{aligned}
\int \operatorname{tr} T(g, h(s)) d \mu(s) & =\int \operatorname{tr}\left(T_{g}^{\prime}, h(s)\right) d \mu(s)=\operatorname{tr}\left(T_{g}^{\prime},(P) \int h(s) d s\right) \\
& =\operatorname{tr} T\left(g,(P) \int h(s) d s\right),
\end{aligned}
$$

which means that $\left(g,(P) \int h(s) d s\right)$ is the Pettis integral of $(g, h(s))\left(T^{\prime}\right.$ is as in Lemma 2). But this simply means that $h(s)$ is $P$-integrable and $P(h)=$ (P) $\int h(s) d s$.

5. We are now in a position to generalize Theorem 2 of $\$ 30$ in [5]. Let $G$ be a locally compact group; consider its group algebra $L^{1}(G)$. As it was defined above, a positive $A$-functional on $L^{1}(G)$ is a linear mapping $\varphi: L^{1}(G) \rightarrow A$ which has an extension $\varphi^{\prime}$ to

$$
L^{1}(G) e=\left\{\lambda e+a \mid a \in L^{1}(G), \lambda \text { complex }\right\}
$$

and the extension $\varphi^{\prime}$ is a positive $A$-functional on $L^{1}(G) e$. Positive definite $A$-function was defined in [7] as a mapping $p: G \rightarrow \tau(A)$ such that $\sum_{i, j} a_{i}^{*} p\left(t_{i}^{-1} t_{j}\right) a_{j} \geqq 0$ for $t_{i} \in G, a_{i} \in A$.

THEOREM 2. If $p: G \rightarrow \tau(A)$ is a continuous (with respect to the norm ( )) positive definite A-function then the mapping $t \rightarrow x(t) p(t)$ is both Pettis and P-integrable for each $x \in L^{1}(G)$ and the function $\varphi(x)=\int x(t) p(t) d t$ is a positive A-functional on $L^{1}(G)$. Conversely each positive A-functional on $L^{1}(G)$ is of this form.

Proof. Let $p$ be a continuous positive definite $A$-function on $G$. According to Theorem 1 of [7] there exists a continuous representation $t \rightarrow U_{t}$ of $G$ by $A$-unitary operators on a Hilbert module $H$ and a member $f_{0}$ of $H$ such that $p(t)=\left(U_{t} f_{0}, f_{0}\right)$ for all $t \in G$. But then the mapping $t \rightarrow V_{t}=U_{t}^{*}$ is also a continuous representation of $G$ by $A$-unitary operators and so it follows from Theorem 2 of [7] that the mapping $x \rightarrow T x=\int_{G} x(t) V_{t} d t$ is a regular *-representation of $L^{1}(G)$. 
Now note that the mapping $t \rightarrow x(t) V_{t} f$ is Pettis integrable for each $f \in H$ and $(P) \int_{G} x(t) V_{t} f d t=\left(\int x(t) V_{t} d t\right) f$ for all $f \in H$ (the last integral here has the same meaning as the corresponding integral in Theorem 2 of [7]). It follows then from Lemma 3 above that

$$
\begin{aligned}
\left(f_{0}, T x f_{0}\right) & =\left(f_{0},\left(\int_{G} x(t) V_{t} d t\right) f_{0}\right)=\left(f_{0}, P\left(x(t) V_{t} f_{0}\right)\right) \\
& =(P) \int_{G} x(t)\left(f_{0}, V_{t} f_{0}\right) d t=(P) \int_{G} x(t)\left(U_{t} f_{0}, f_{0}\right) d t \\
& =(P) \int_{G} x(t) p(t) d t=\varphi(x)
\end{aligned}
$$

for all $x \in L^{1}(G)$. If we extend $T$ to $L^{1}(G) e$ by setting $T(\lambda e+x)=$ $\lambda I+T x$ we see immediately that $\varphi$ has an extension $\varphi^{\prime}$ defined by $\varphi^{\prime}(\lambda e+x)=\left(f_{0}, T(\lambda e+x) f_{0}\right)=\lambda\left(f_{0}, f_{0}\right)+\left(f_{0}, T x f_{0}\right)=\lambda\left(f_{0}, f_{0}\right)+\varphi(x)$ and that $\varphi^{\prime}$ is a positive $A$-functional on $L^{1}(G) e$.

The converse is also established in a similar manner. If $\varphi$ is a positive $A$-functional on $L^{1}(G)$ then $\varphi$ is of the form $\varphi(x)=\left(f_{0}, T x f_{0}\right)$ for some *-representation $T$ of $L^{1}(G)$ by $A$-linear operators. It follows from Theorem 2 of [7] that there exists a continuous representation $t \rightarrow U_{t}$ of $G$ (by $A$-unitary operators) such that $T x=\int_{G} x(t) U_{t} d t$ for all $x \in L^{1}(G)$. Then $p(t)=\left(U_{t} f_{0}, f_{0}\right)$ is a positive $A$-function and $\varphi(t)=\left(f_{0}, T x f_{0}\right)=$ $\int_{G} x(t)\left(f_{0}, U_{t} f_{0}\right) d t=\int_{G} x(t) p(t)$ for all $x \in L^{1}(G)$.

\section{REFERENCES}

1. W. Ambrose, Structure theorems for a special class of Banach algebras, Trans. Amer. Math. Soc. 57 (1945), 364-386. MR 7, 126.

2. E. Hille and R. S. Phillips, Functional analysis and semi-groups, Amer. Math. Soc. Colloq. Publ., vol. 31, rev. ed., Amer. Math. Soc., Providence, R.I., 1957. MR 19, 664.

3. L. H. Loomis, An introduction to abstract harmonic analysis, Van Nostrand, New York, 1953. MR 14, 883.

4. G. W. Mackey, Commutative Banach algebra, Lecture notes, Harvard University, 1952; Livraria Castelo, Rio de Janeiro, 1959.

5. M. A. Naimark, Normed rings, GITTL, Moscow, 1956; English transl., Noordhoff, Groningen, 1959. MR 19, 870; 22 \#1824.

6. P. P. Saworotnow, A generalized Hilbert space, Duke Math. J. 35 (1968), 191-197. MR 37 \#3333.

7. - Representation of a topological group on a Hilbert module, Duke Math. J. 37 (1970), 145-150. MR 41 \#7442.

8. P. P. Saworotnow and J. C. Friedell, Trace-class for an arbitrary $\mathrm{H}^{*}$-algebra, Proc. Amer. Math. Soc. 26 (1970), 95-100.

9. P. P. Saworotnow, Trace-class and centralizer of an $H^{*}$-algebra, Proc. Amer. Math. Soc. 26 (1970), 101-104.

Department of Mathematics, The Catholic University of America, Washington D.C. 20017 